

\section{ÍNDICE}

\section{ÁMBITOS PERSONALES PERSONALÁMBITOS}

Crisis de Venezuela: Análisis y perspectivas según los titulares de la prensa argentina, española y china

Venezuela's crisis: Analysis and perspectives in the headlines of Argentine, Spain and China press

Hui Feng Liu

Tratamiento informativo de la violencia de género: asesinatos de mujeres. Análisis de la agencia EFE

Informative treatment of gender violence: murders of women. Analysis of the press agency EFE Rosa Rodríguez Cárcela, Agustín López Vivas

MONOGRAFICOS MONOGRAPHS

Presentación Monográfico. El universo transmedia de los medios de comunicación universitarios: acción dentro y fuera del aula en la sociedad postdigital

Antonia Isabel Nogales-Bocio, Ángels Álvarez villa

El papel transformador de la radio universitaria en materias teóricas ajenas a la comunicación

The transformative role of college radio in theoretical subjects outside communication

Miguel Ángel Díaz Monsalvo

La radiodifusión universitaria: acción discursiva radiofónica para la divulgación de la ciencia University Radio Broadcasting: Radiophonic Discursive Action for the Science Popularization Jorge Sadi Durón, Joel Zapata Salazar

El uso corporativo de Instagram en las universidades privadas españolas. Estudio comparativo de treinta y cinco universidades

The corporate use of Instagram in spanish private universities. Comparative analysis of thirty-five private universities 
La radio universitaria como herramienta de inclusión social: OndaCampus en contextos como la cárcel y barrios desfavorecidos

The university radio as a tool for social inclusion: OndaCampus in contexts like the prison and disadvantaged neighborhoods

Leonor Real Adame, Daniel Martín-Pena, Macarena Parejo Cuéllar

Hacer radio universitaria en la era de YouTube: uso de la plataforma de vídeos a demanda en el contexto mexicano

Make college radio in the age YouTube: use of video on demand platform in the Mexican context Marina Vázquez Guerrero

\section{ARTÍCULOS ARTICLES}

O impresso e o digital nos modelos de negócios de jornais locais: uma análise do Sermos Galiza

The press and digital in business models from local media: Analysis of newspaper Sermos Galiza

Giovanni Ramos

Publicidad y cáncer en la prensa escrita (1903-1912)

Advertising and cancer in the written press (1903-1912)

Laura Almudéver-Campo, Ramón Camaño-Puig

Game rules vs. fandom. How Nintendo's Animal Crossing fan-made content negotiates the videogame meanings

Las reglas del juego vs. el fandom. Cómo el contenido hecho por fans de Nintendo Animal Crossing negocia los significados del videojuego

Jose A. Moreno

La comedia de situación y su análisis textual: evolución de los elementos constructivos del formato

The sitcom and its textual analysis: evolution of the constructive elements of the format

Darío Martín Sánchez

Análisis del discurso emocional de Donald Trump en la campaña electoral de $\mathbf{2 0 1 6}$ Analysis of Donald Trump's emotional speech on the 2016 election campaign 
Innovar, comunicar y transformar (en) la Universidad

Innovate, communicate and transform (at) the University

María Sofía Bernat

288-292

Transparencia en los medios: Un requisito imprescindible para medir la rentabilidad social en radio y televisión

Transparency in the media: An essential requirement to measuresocial profitability in radio and television

Amanda Salazar

Los estudios feministas en comunicación: representación de las mujeres en la revolución tecnológica

Feminist studies in communication: representation of women in the technological revolution 


\title{
Los estudios feministas en comunicación: representación de las mujeres en la revolución tecnológica
}

Feminist studies in communication: representation of women in the technological revolution

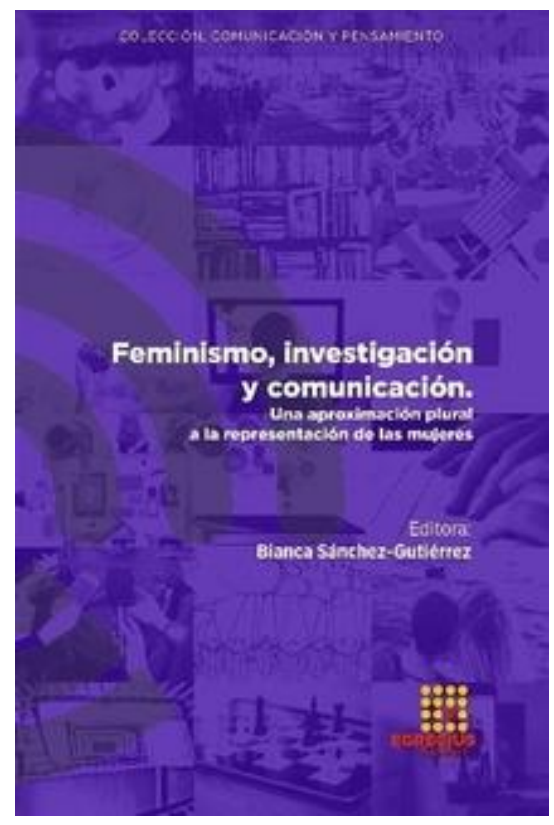

\author{
Feminismo, investigación y \\ comunicación: Una aproximación plural a
}

la representación de las mujeres

Bianca Sánchez-Gutiérrez

Egregius, España, 2018

146 páginas

Reseña por Sara Rebollo-Bueno

DOI: http://dx.doi.org/10.12795/Ambitos.2020.i47.16 
La época actual está marcada por los grandes avances tecnológicos, de hecho, algunos autores la denominan como la revolución tecnológica. En esta revolución, las redes sociales tienen un papel fundamental, pues han conseguido dar un giro a la comunicación y convertirse en herramientas imprescindibles. La inmediatez y la creación de canales directos son características de estas nuevas herramientas, las cuales están siendo principales a la hora de realizar la comunicación de diferentes movimientos sociales. Entre estos, se encuentra la llamada Cuarta Ola del Feminismo, otra gran revolución que se está viviendo en nuestros días.

Ante este nuevo panorama mediático y social se hace imprescindible otorgar perspectiva feminista a las investigaciones en comunicación, pues la repercusión social de ambas revoluciones, la tecnológica y la feminista, así lo precisan. Con este objetivo, asegura la editora Bianca Sánchez-Gutiérrez, nace Feminismo, investigación y comunicación: una aproximación plural a la representación de las mujeres, cuyo origen es la selección de investigaciones que participaron en el simposio "Feminizando la Red: Tendencias comunicativas y perspectiva de género" del III Congreso Internacional de Comunicación y Pensamiento: Generación Smartphone, Comunicación Móvil, celebrado en Sevilla en abril de 2018

Este libro aporta una perspectiva amplia y rigurosa acerca de las investigaciones feministas en comunicación. Cuenta con siete capítulos y una introducción previa. El primer capítulo, cuya autora es Mariché Navío Navarro, realiza un estudio sobre las interacciones online más compartidas sobre dos series de ficción: The Sinner y Alias Grace, obteniendo resultados en cuanto a las críticas vertidas en páginas webs especializadas en productos audiovisuales sobre el rol de la mujer en ambas series. Por otro lado, en el tercer capítulo, Inmaculada Sánchez-Labella Martín, analiza la autorrepresentación de las modelos curvy en Instagram, consiguiendo sacar conclusiones tan contundentes como la existencia de reivindicación en Instagram con el objetivo de normalizar la belleza plus-size.

En el capítulo tercero, El desarrollo del fútbol femenino en el entorno digital: análisis de los contenidos publicados por los clubes en las web oficiales, se expone un análisis de contenido sobre las comunicaciones que realizan los clubes de fútbol. Gracias a la investigación de Rafael Cano Tenorio se confirma la existencia de desigualdades en la comunicación de los clubes femeninos en comparación con los masculinos, dando paso a nuevas líneas de investigación sobre el objeto de estudio. Lola Sanjuán Jurado y Cristina García Carrera son las autoras del cuarto capítulo de la obra, quienes no solo exponen la importancia de la inclusión de las mujeres y los movimientos en los que participan en la agenda mediática, sino que aportan recomendaciones y tácticas para que los medios cumplan con su función social y cambien su perspectiva hacia una visión feminista. El quinto capítulo, escrito por Clara Pazos Polo y David Polo Serrano, hace un recorrido por la documentación existente acerca de la figura de la mujer durante el nazismo, pues a pesar de la escasa bibliografía acerca de este objeto de estudio, los 
autores han conseguido obtener importantes conclusiones como, por ejemplo, el doble papel que tenían las mujeres en el Régimen Hitleriano, ya que no estaban solamente relegadas a ser madres sino que también eran trabajadoras del propio Régimen.

Regla Ismaray Cabreja Piedra, en el sexto capítulo, hace un acercamiento a la realidad que viven las mujeres en Latinoamérica, dado que es la región que cuenta con un mayor número de casos de violencia de género. La autora se centra en Cuba y hace un análisis minucioso de los medios digitales de la isla, así como de la situación jurídica del país con respecto a la violencia de género. El último capítulo de la obra, El activismo social feminista: La violencia de género como centro mediático, de Lilia Carpio Jiménez, Kruzkaya Ordóñez y Abel Suing, pone el foco en los movimientos feministas surgidos en redes sociales, como el Time's Up, pues pretendían realizar ruido mediático a través de estas plataformas. Asimismo, los autores exponen sus resultados en perspectiva, comparándolos con otros movimientos con el mismo o parecido objetivo, como puede ser el Me Too.

Feminismo, investigación y comunicación: una aproximación plural a la representación de las mujeres es una obra que consigue dar al lector una base sólida, estructurada y rigurosa acerca de los estudios académicos feministas en comunicación. De hecho, puede afirmarse que este libro no solo tiene una finalidad de difusión científica, sino que tiene un compromiso social, pues apoya la lucha social que conlleva el Feminismo por la búsqueda de la igualdad real entre hombres y mujeres. 